\title{
The Influence of Personality Type D on Cardiovascular Prognosis in Patients After Coronary Artery Bypass Grafting: Data from a 5-Year-Follow-up Study
}

\author{
Olga Igorevna Raykh ${ }^{1}$. Alexei Nikolayevich Sumin ${ }^{2}\left[\right.$ Ekaterina Victorovna Korok ${ }^{3}[$
}

Accepted: 18 April 2021 / Published online: 6 May 2021

(c) International Society of Behavioral Medicine 2021

\begin{abstract}
Background Type D personality is accompanied by a set of negative behavioral patterns: low physical activity, high levels of psychological distress, low adherence to treatment. However, studies regarding predictive value of the type D personality remain inconclusive: the results varied depending on the examined cohort, age, and ethnicity. The aim of the study was to evaluate the influence of type $\mathrm{D}$ personality on the 5-year prognosis in patients undergoing coronary artery bypass grafting (CABG) in Russian population.

Methods The study included 602 patients with stable coronary artery disease (490 males, $57.7 \pm 7.3$ years) who had received CABG and were divided into two groups: patients with type D personality $(n=134)$ and patients without type $\mathrm{D}(n=468)$. The risk of fatal and nonfatal events within 5 years after CABG was assessed.

Results There was no difference in total mortality in patients with type $\mathrm{D}$ and without type $\mathrm{D}(7.9 \%$ and $7.7 \%$, respectively) over the 5-year period. The absence of cardiac events was detected much less frequently in patients with type D (28\%) compared with patients without type $\mathrm{D}(82 \% ; p=0.021)$. Multivariate analysis found independent association between the unfavorable outcome and presence of diabetes mellitus $(p=0.021)$, type $\mathrm{D}$ personality $(p=0.039)$, and multifocal atherosclerosis $(p=0.033)$ regardless of gender, age, previous myocardial infarction, and stroke.

Conclusions Type D patients had a greater risk for cardiac events over 5 years after CABG compared with non-type D patients. Obtained data indicates that it is reasonable to consider personality type while detecting patients at risk of development of stress induced cardiac complications after CABG.
\end{abstract}

Keywords Personality type D · Prognosis - Coronary artery bypass grafting

Alexei Nikolayevich Sumin

an_sumin@mail.ru; sumian@kemcardio.ru

Olga Igorevna Raykh

raihoi@inbox.ru

Ekaterina Victorovna Korok

korok-82@mail.ru

1 Researcher of Laboratory of Circulation Pathology, FSBI Research Institute of Complex Problems of Cardiovascular Disease, Kemerovo, Russian Federation

2 Head of Department of Polyvascular Disease, FSBI Research Institute of Complex Problems of Cardiovascular Disease, Kemerovo, Russian Federation

3 Researcher of laboratory of Circulation Pathology, FSBI Research Institute of Complex Problems of cardiovascular disease, Kemerovo, Russian Federation

\section{Introduction}

Over the recent years, the concept of patient-centered medicine has evolved from a theoretical principle into a basic dimension of high-quality medical care. In modern medical practice, the patient's vision, participation in joint decision-making, and focus on the results reported by patients are becoming increasingly important, which is supported by increased attention to the patient-oriented approach in research [1]. Orientation to the patient is a multidimensional concept, one of the dimensions of which is the idea of "Patient as a person," meaning that, depending on patient's needs, the effect of function impairment can have different consequences. Thus, the patient's current life situation is another factor that has to be taken into account. The patient-oriented approach for cardiovascular diseases includes the patient's participation in making decisions on treatment methods and assessment of his satisfaction with the treatment 
[2], i.e., how much his quality of life is changing. And with this approach, we inevitably encounter the personality characteristics that can affect the patient's state of health regardless of the success of the treatment. Assessment of the patient's personality encompassed the individuality of each patient in different cases of the disease [1]. Expectedly, the clinicians keep searching for a convenient assessing tool for the personal traits of patients.

The concept of type D (distressed) personality developed by the Prof. Denollet and colleagues [3, 4] looked quite suitable for this purpose [5]. Type D personality consisted of a combination of negative affectivity in stressful situations (negative affectivity (NA)) and the suppression of its manifestations in social interactions (social inhibition (SI)). Importantly, the determination of type D personality was carried out using the DS14 questionnaire, which only contains 14 questions and is easily applicable in a clinical setting [4]. It is advisable to take type D personality into account in patient-oriented approaches, since such individuals are characterized by lower quality of life [6, 7], a tendency to develop depressive reactions [8], decreased compliance to treatment $[9,10]$, and implementation of medical recommendations [11]. In our opinion, even this information about the type D personality makes using it in patient-oriented approaches to treatment of cardiovascular patients reasonable. Type $\mathrm{D}$ personality is one of the nine psychosocial risk factors that were identified by the European Society of Cardiology as independent risk factors for incidence and prognosis of heart disease [12]. In comparison, other personality construct mentioned as a risk factor is hostility/anger, while other risk factors are mood and stress- and social support related. At the same time, the Big Five personality traits are not considered psychological risk factors in these guidelines. Besides, personality Type D on the one hand, it is negatively associated with the quality of life of patients [6, 7], which one of the goals of the patientcentered approach. On the other hand, personality type D adversely affects doctor-patient communication. So, in the study of Schiffer et al., patients with type D personality were less likely to report the symptoms to their cardiologist/nurse in contrast with patients with a non-type-D personality [11].

Moreover, in the initial studies, coronary artery disease (CAD) patients with type D personality had a worse prognosis compared to patients without type D [3]. Accordingly, type $\mathrm{D}$ personality was considered as one of the chronic psychological stress factors in development of cardiovascular diseases [13], and since 2012, type D personality has been included in the European Cardiovascular Prevention guideline as a risk factor to screen for [12]. However, at the turn of the decade, three large studies revealed no prognostic significance of personality Type $\mathrm{D}$ for mortality in patients with heart failure $[14,15]$ and in cardiac patients from a German cohort study [16]. These results were inconsistent with both the results of primary studies with type D personality [3] and subsequent data from different groups of researchers [17-20]. These conflicting results can be due to several reasons, one of which is methodological problems, for example, the dichotomy of NA and SI in determining the identity of the type D personality or inappropriate statistics [21].

To solve this problem, it was recommended to evaluate personality type $\mathrm{D}$ not as a dichotomous variable, but separately on the NA and SI scales, as continuous variables, and their interaction to identify the "type D effect" [22]. In some subsequent articles, this approach for assessing the personality type D and its "effect" was used [23], but the results remained ambiguous. For example, when monitoring a group of patients with cardiovascular disease for up to 5 years, it was found that the interaction of $\mathrm{NA} \times \mathrm{SI}$ was independently associated with major adverse cardiac event (MACE); however, SI and NA were not associated with MACE separately [23]. Contrariwise, the 2-group approach in the study of Dulfer et al. revealed that people with type $\mathrm{D}$ personality had a larger all-cause mortality than people without type $\mathrm{D}$ personality $(\mathrm{OR}=1.58,95 \%=1.22$, $2.03)$, which was not noted with the continuous interaction approach $(\mathrm{OR}=0.95,95 \%$ confidence interval $[\mathrm{CI}]=0.78$, 1.17) [17]. Similar difference in clinical indicators between the dichotomous assessment of type D and the interaction of continuous variables of NA and SI was observed in studies by Wang et al. [24, 25] and Williams et al. [26]. Apparently, this approach confuses the situation with the assessment of the "type D effect" even more, increasing the heterogeneity of the obtained results, complicating the initial concept of type D significantly and making its practical application difficult. Therefore, in the present study, we used a dichotomous assessment of type D personality, in particular, since our previous studies showed the effectiveness of just such an approach to identifying the main "effects of type D" in the Russian population.

According to the results of a meta-analysis of 12 studies that examined the prognostic effect of a type D personality, type $\mathrm{D}$ personality increased risk of mortality in CAD patients significantly, while patients with heart failure did not have an increased risk of mortality [17]. An additional analysis of the data in CAD patients [20] showed that the heterogeneity of the data on the prognostic effect for type $\mathrm{D}$ personality can be explained by the stage of the disease (CAD or heart failure), research endpoint (mortality from all causes against cardiac events and cardiac death), as well as the age of patients [20]. Since the initial cohort of patients was limited to only two countries (the Netherlands and Germany), the question arises whether ethnic, geographic, and socio-economic factors can influence the prognosis for type D personality (the COVID-19 pandemic clearly showed the significance of these factors [27]).

In this regard, the results of a study by Kupper et al. are interesting [28], in which the intercultural significance of type D constructs was studied in 22 countries as a part of the HeartQoL international project. On one hand, that study 
established intercultural equivalence of measurements for type D personality at all levels of measurement, since the configuration of the factor element, factor loading, and error structure, as well as gender and diagnostic subgroups, did not differ between countries. On the other hand, a difference in prevalence of the type $\mathrm{D}$ personality was noted depending on the region: it is more common in southern (37\%) and eastern (35\%) European countries compared to Northern (24\%) and Western European and English-speaking ones (both 27\%) $(p<0.001)$. Therefore, studies on the prognostic value of personality type $\mathrm{D}$ in different regions remain relevant. It was previously shown that the Russian version of DS14 coincides with the English version in terms of reliability and internal structure, the validity of the design is confirmed by the results of correlation analysis [29].

According to our previous data, in the population sample of Russian patients with type D personality, there is more pronounced calcification of the coronary arteries [30], patients with type D have lower adherence to treatment [31], and multifocal atherosclerosis is more often detected in type $\mathrm{D}$ personality [32]. These factors are associated with a poor prognosis in various cardiovascular diseases. All this suggests that type D personality will also negatively affect the prognosis in cardiac patients. It has previously been shown that cardiovascular complications develop more often within 1 year after CABG compared with patients without type $\mathrm{D}$ personality [19], but these data require confirmation with a longer follow-up period in order to be consistent with previous studies of the predictive value of personality type D [16]. Accordingly, the aim of our study was to assess the influence of a predisposition to psychological distress on long-term results in patients undergoing coronary artery bypass grafting $(\mathrm{CABG})$ in the Russian population.

\section{Methods}

\section{Participants and Procedure}

This observational prospective cohort study was carried out in the FSBI "Research Institute of Complex Problems of cardiovascular disease" among CAD patients before coronary artery bypass surgery (CABG) in the period from 2010 to 2011. Patients were included in the study with the planned nature of CABG surgery and if they were able to complete the DS14 questionnaire. Patients were excluded in case of emergency surgery and the impossibility of a complete preoperative examination, as well as if they had cognitive impairment that interfered with filling out questionnaire. In total, 709 patients with CAD (132 (18.5\%) females and 577 (81.5\%) males, age $57.7 \pm 7.3$ years) were included in the study. The study protocol was approved by the Local Ethics Committee of the Federal State Budgetary Institution Research Institute for Complex Issues of Cardiovascular
Diseases, and was performed in accordance with the declaration of Helsinki as revised in 1989. Patients were included in the study after they provided written informed consent.

\section{Study Parameters and Instruments}

\section{Socio-demographic and Clinical Characteristics}

Before the CABG, all patients underwent coronary angiography, echocardiography, color duplex examination of the carotid vessels, and Doppler study of lower extremity arteries. Baseline demographic and clinical data, including cardiovascular risk factors such as hypertension, obesity, smoking status, diabetes mellitus and dyslipidemia, history of myocardial infarction, stroke, carotid endarterectomy, signs of multifocal atherosclerosis, atrial fibrillation, manifestations of heart failure, left ventricular ejection fraction, and the risk of surgery on the EuroSCORE scale were obtained from the patient's medical records. The details of coronary bypass surgery were documented, such as duration of aortic clamping, time of bypass, total number of bypass grafts, surgery without bypass, and the presence of perioperative complications (myocardial infarction, stroke, atrial fibrillation, heart failure, acute renal failure, pneumonia, multiple organ failure syndrome). Preoperative and perioperative data was obtained from the cardiac research databases at our institution and reviewed in the hospital charts.

\section{Type D Personality}

The evaluation of the psychological status was carried out using questionnaire DS14, in the preoperative period and postoperative period, validated in Russian [29]. The DS14 questionnaire comprises two subscales: negative affectivity (NA) and social inhibition (SI), containing seven questions each. To express agreement/disagreement with each item, a 5-point Likert scale from 0 (false) to 4 (true) was used. Hence, the total scores for NA and SI subscales ranges from 0 to 28 . If the score was $\geq 10$ points on both subscales, type D personality was diagnosed. The DS-14 is a valid measure of negative affectivity and social inhibition in the Russian general population [29]; in this study, Cronbach's alpha for NA was 0.78 , for SI 0.74 , which confirms the adequacy of intrinsic structure of Russian version of DS14.

\section{Follow-up}

Patients included in the study for 5 years were observed by a cardiologist in their place of residence. The follow-up was performed 5 years after the surgery by personal visits, telephone surveys, and analyzing outpatient medical records. The primary endpoint included major adverse cardiovascular events (MACE-total mortality, nonfatal MI, and nonfatal 
stroke), as well as re-revascularization and hospitalization due to relapse or progression of angina pectoris. Secondary endpoints included MACE alone (total mortality, nonfatal MI, and nonfatal stroke).

\section{Statistical Analyses}

The standard software packages "STATISTICA 8.0" and SPSS 17.0 were used for statistical processing. All data are presented as the median and quartiles $(\mathrm{Me} \pm \mathrm{Q})$ or frequencies and percentages. Differences in continuous variables between the two groups were assessed using the Mann-Whitney $U$ test. Comparisons of patients were performed with Student's $t$ test for continuous variables and the $\chi^{2}$ test or Fisher's exact test for categorical variables. The Kaplan-Meier method was used to calculate survival free from the combined end-point (primary endpoint) or MACEs (secondary endpoint). Univariate and multivariable logistic regression analysis was used to calculate odds ratios (OR) and thus determine the independent predictors of a combined endpoint (primary endpoint). As independent variables, the model included variables characterizing the condition of patients before coronary bypass surgery (age, gender, body mass index, arterial hypertension, diabetes, dyslipidemia, multifocal atherosclerosis, smoking, left ventricular ejection fraction, atrial fibrillation, risk of surgery on a scale EuroSCORE, history of myocardial infarction, stroke, personality type D). Age, sex, and variables with $p<0.1$ on univariate analysis were entered into a multivariate analysis. Preliminarily, the identification of possible correlations between the putative predictors was carried out; then, several regression models were formed taking into account the identified correlations. Adjusted hazard ratios were assessed in a multivariate model using variables that were retained in the final model by the backward stepwise deletion method. To construct a multivariate analysis models taken into account paired correlation coefficient, the OR and $95 \%$ CI were given. To analyze the synergistic interaction approach in predictive value of type $\mathrm{D}$ personality on primary endpoint development, the binomial logistic regression was used. Continuous NA and SI scores were computed into z-scores. These variables, together with the interaction term $(\mathrm{zNA} \times \mathrm{SI})$, were entered in the Forward stepwise binomial logistic regression model to examine the influence of type $\mathrm{D}$ personality (continuous) on primary endpoint development. $p<0.05$ was considered statistically significant.

\section{Results}

\section{Study Participants}

Forty-four cases of death were identified in 5 years after surgery. For various reasons, we were not able to contact 23 patients (3.2\%), and 40 patients (5.6\%) who could not come for examination were interviewed by phone (Fig. 1). As a result, upon the expiration of a 5-year period, the patients were divided into two groups: patients with type $\mathrm{D}(n=134)$ and patients without type $\mathrm{D}(n=468)$. All patients in the postoperative period underwent complex physical, instrumental examination and assessment of personality type $\mathrm{D}$. The median follow-up duration was 38.0 months (range, 0.3 to 63.9 months).

\section{Baseline Characteristics}

Clinical characteristics are presented in Table 1. There was no statistically significant difference between two groups in age, gender, prevalence of "old" myocardial infarction (MI), smoking, and other demographical variables. Herewith the groups were statistically significantly different in prevalence of previous stroke, and multifocal atherosclerosis $(p=.011)$. Aspirin, $\beta$-blockers, angiotensin-converting enzyme inhibitors, and statins were administered in both groups with equal frequency $(p>.05)$. Type $\mathrm{D}$ personality was detected in 134 (22.3\%) patients preoperatively, and similar results were found after the 5-year period-123 (22.4\%) patients had type D
Fig. 1 Flowchart of patient selection

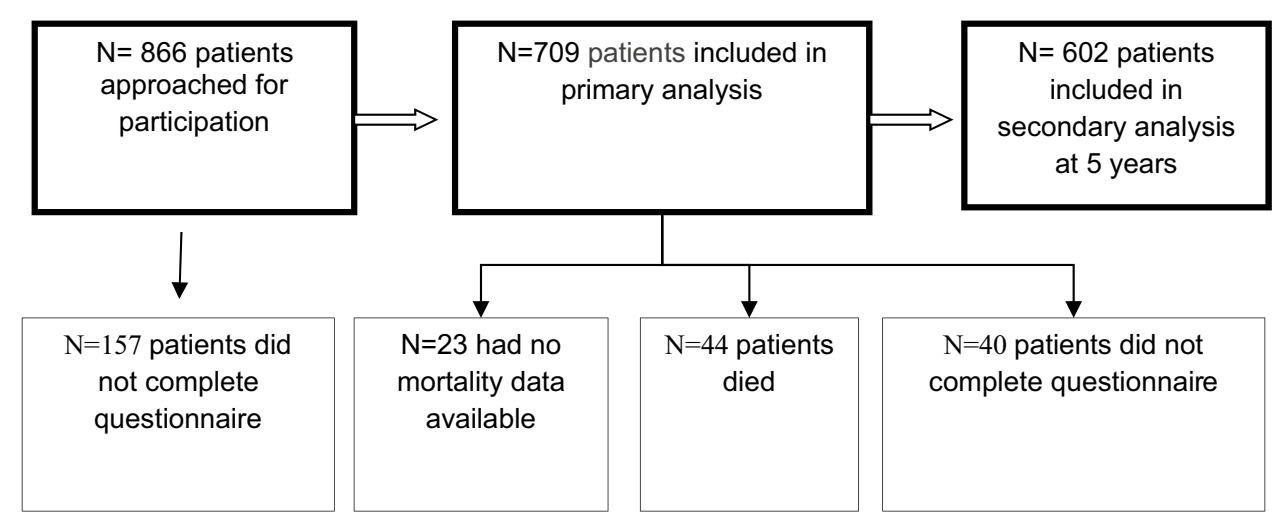


Table 1 Baseline characteristics of the studied population

\begin{tabular}{llll}
\hline Variables & Type D $(n=134)$ & Non-type D $(n=468)$ & $p$ \\
\hline Age, years & $60 \pm 3$ & $58 \pm 4$ & 0.082 \\
Male gender $(\%)$ & 75.2 & 81.8 & 0.122 \\
BMI $\left(\mathrm{kg} / \mathrm{m}^{2}\right)$ & $25.1 \pm 4.2$ & $24.4 \pm 4.0$ & 0.671 \\
Smoker $(n, \%)$ & $110(72.2)$ & $302(67.6)$ & 0.051 \\
Hypertension, yes, $(n, \%)$ & $121(90)$ & $409(87.3)$ & 0.542 \\
Diabetes, yes $(n, \%)$ & $26(19.7)$ & $64(13.7)$ & 0.056 \\
Atherogenic indices & $3.7 \pm 0.8$ & $3.4 \pm 0.9$ & 0.621 \\
Previous myocardial infarction $(n, \%)$ & $85(63.1)$ & $329(70.3)$ & 0.062 \\
Previous stroke $(n, \%)$ & $\mathbf{1 6}(\mathbf{1 2 . 1})$ & $\mathbf{3 2}(\mathbf{6 . 9})$ & $\mathbf{0 . 0 1 1}$ \\
Previous carotid endarterectomy $(n, \%)$ & $6(4.8)$ & $15(3.2)$ & 0.312 \\
Multifocal atherosclerosis $(n, \%)$ & $\mathbf{4 2 ( 3 1 . 4 )}$ & $\mathbf{9 3}(\mathbf{1 9 . 8})$ & $\mathbf{0 . 0 1 1}$ \\
Atrial fibrillation $(n, \%)$ & $18(13.2)$ & $48(10.3)$ & 0.116 \\
Heart failure NYHA II-IV $(n, \%)$ & $108(81.3)$ & $367(78.5)$ & 0.212 \\
Left ventricular ejection fraction $(\%)$ & $55 \pm 10.48$ & $57 \pm 10.06$ & 0.132 \\
Surgical risk by EuroSCORE & $2.2 \pm 0.10$ & $1.9 \pm 0.12$ & 0.091 \\
\hline
\end{tabular}

BMI body mass index, NYHA New York Heart Association heart failure scale, The entries in italics indicate borderline statistical significance of differences between groups $(p<0.1)$, the entries in bold indicate significant differences $(p<0.05)$ between groups personality $(p>.05)$, which shows a remarkable stability of personality characteristics, having no changes in $96 \%$ over a 5-year period. Intraoperative and perioperative patient characteristics are summarized in Table 2. As seen in Table 2, no statistically significant differences between groups in prevalence of early complications or in-hospital mortality were found. At the same time, the frequency of the following complications was slightly higher in the group with type D compared with the group without type D: atrial fibrillation, acute renal failure, and pneumonia (however, the trends remained statistically insignificant).
Thus, the mortality was $2.8 \%$ and $2.6 \%$, stroke occurred in $1.5 \%$ and $0.8 \%$, and MI occurred in $0.7 \%$ and $0.6 \%$ of the patients, respectively.

\section{Follow-up Outcomes}

Overall mortality and cardiac mortality in patients of both groups did not differ over long-term period after CABG surgery, remaining at $7.9 \%$ and $7.7 \%$, and $2.8 \%$ and $2.6 \%$, respectively. The most prevalent cause of patients' death was heart failure, which occurred in $15.2 \%$ patients, as an
Table 2 Comparison of intraoperative and perioperative variables in patients undergoing coronary artery bypass grafting

\begin{tabular}{llll}
\hline Variables & Type D $(n=134)$ & Non-type D $(n=486)$ & $p$ \\
\hline CABG with CPB $(n, \%)$ & $109(81.3)$ & $387(79.6)$ & 0.482 \\
CABG off-pump $(n, \%)$ & $27(19.8)$ & $82(16.8)$ & 0.771 \\
CABG + catheter ablation $(n, \%)$ & $10(7.7)$ & $21(4.4)$ & 0.442 \\
Duration of CPB, min & $99.2 \pm 4.9$ & $97.9 \pm 5.0$ & 0.211 \\
Cross-clamp time, min & $61.7 \pm 3.1$ & $54.4 \pm 2.0$ & 0.454 \\
Total number of grafts & $2.31 \pm 0.06$ & $2.29 \pm 0.06$ & 0.181 \\
Perioperative myocardial infarction $(n, \%)$ & $1(0.7)$ & $3(0.6)$ & 0.423 \\
Stroke $(n, \%)$ & $2(1.5)$ & $4(0.8)$ & 0.522 \\
Atrial fibrillation $(n, \%)$ & $29(21.9)$ & $71(14.6)$ & 0.051 \\
Heart failure $(n, \%)$ & $10(7.7)$ & $34(6.9)$ & 0.812 \\
Acute renal failure $(n, \%)$ & $7(5.5)$ & $14(2.9)$ & 0.091 \\
Pneumonia $(n, \%)$ & $13(9.9)$ & $37(7.6)$ & 0.186 \\
MODS $(n, \%)$ & $4(3.3)$ & $9(1.9)$ & 0.591 \\
\hline
\end{tabular}

$C A B G$ coronary artery bypass grafting, $C B P$ cardiopulmonary bypass, $M O D S$ multiple organ dysfunction syndrome, The entries in italics indicate borderline statistical significance of differences between groups $(p$ $<0.1)$, the entries in bold indicate significant differences $(p<0.05)$ between groups 
Table 3 Comparison of postoperative variables in patients undergoing elective coronary artery bypass grafting

\begin{tabular}{llll}
\hline Variables & Type D $(n=134)$ & Non-type D $(n=468)$ & $p$ \\
\hline Stroke $(n, \%)$ & $17(12.4 \%)$ & $56(11.9 \%)$ & 0.142 \\
Myocardial infarction $(n, \%)$ & $11(8.2 \%)$ & $50(10.8 \%)$ & 0.251 \\
Rates of hospitalizations $(n, \%)$ & $35(26.1 \%)$ & $99(21.2 \%)$ & 0.053 \\
$\begin{array}{l}\text { Rates of hospitalizations for cardiovascu- } \\
\quad \text { lar diseases }(n, \%)\end{array}$ & $\mathbf{9 ( 2 5 . 7 \% )}$ & $\mathbf{2 0}(\mathbf{2 0 . 2 \%})$ & $\mathbf{0 . 0 4 1}$ \\
Repeated revascularization $(n, \%)$ & $25(18.7)$ & $12(2.6)$ & 0.057 \\
\hline
\end{tabular}

The entries in italics indicate borderline statistical significance of differences between groups $(p<0.1)$, the entries in bold indicate significant differences $(p<0.05)$ between groups outcome of acute coronary circulatory disorders (13.1\%), progression of coronary atherosclerosis, stent thrombosis $(0.9 \%)$, and/or native vessels thrombosis (1.9\%). Adverse cardiac events in patients occurred predominantly during the first 3 years after CABG surgery.

During follow-up, 61 of patients developed MI (10.2\%), 73 suffered a stroke (12.2\%) with no intergroup difference for prevalence of these events. Indications for revascularization were determined in 31 patients of both groups $(5.2 \%)$ in a 5-year period. In 18 patients, reoperation was necessary because of graft dysfunction leading to return of angina. In 9 cases, revascularization was indicated due to progression of atherosclerosis, confirmed by coronary angiography. Four patients did no undergo intervention for various reasons.

Most patients indicated for revascularization were found to have type D personality (25 patients of 31 ); however, the small sample size led to statistical insignificance of this result (Table 3 ).

In long term, functional class (FC) I-II angina frequency was significantly less prevalent in the group with type D compared to the group without it (77\% vs. 83\%) $(p=.022)$, herewith the FC III-IV angina was significantly more prevalent ( $13 \%$ vs. $6.4 \%, p=.041)$. The study did not detect any difference in prevalence of heart failure (Fig. 2).

A significant difference was determined assessing degrees of freedom from primary endpoint: $28 \%$ with type D vs.
$82 \%$ in the group without type D ( $p=.021)$ (Fig. 3). At the same time, freedom from the MACE showed statistically insignificant difference $(65.5 \%$ vs. $62.8 \%, p=.611)$ in the study groups (Fig. 4).

Multiple logistic regression analysis revealed that the following parameters had an independent effect on the relative odds of the combined endpoint: multifocal atherosclerosis (OR 3.60; 95\% CI 0.95-11.34) $(p=.011)$, hyperlipidemia (OR 2.89; 95\% CI 1.45-4.24) $(p=.025)$, diabetes mellitus (DM) (OR 2.92; 95\% CI 1.07-8.81) $(p=.041)$, and type D personality (OR 2.17; 95\% CI 0.91-4.61) $(p=.042)$. Multivariate analysis confirmed (irrespective of sex, age, MI, stroke) such parameters as presence of DM (OR 3.25; 95\% CI 1.25-6.24; $p=.021$ ), type D personality (OR $1.44 ; 95 \%$ CI $1.21-1.81$, $p=.039$ ), and multifocal atherosclerosis (OR 3.61; 95\% CI 1.32-6.24, $p=.033$ ) being independent predictors for combined endpoint (Table 4).

The stepwise binary logistic regression model included continuous z-values on the NA and SI scales (zNA and $\mathrm{zSI}$ ), as well as their interactions (zNA*zSI) (Supplementary Tables 1 and 2). All these indicators revealed the significant association with the primary endpoint development: for zNA $\operatorname{Exp}(B)=1.712, p<.001$; for $\mathrm{zSI}-\operatorname{Exp}$ $(B)=1.296, p=.013$; and for $\mathrm{zNA}^{*} \mathrm{zSI}-\operatorname{Exp}(B)=1.560$, $p<.001$.
Fig. 2 CHD clinical course in the long-term period after CABG. CCS Canadian Cardiovascular Society angina scale, NYHA New York Heart Association heart failure scale, FC functional class

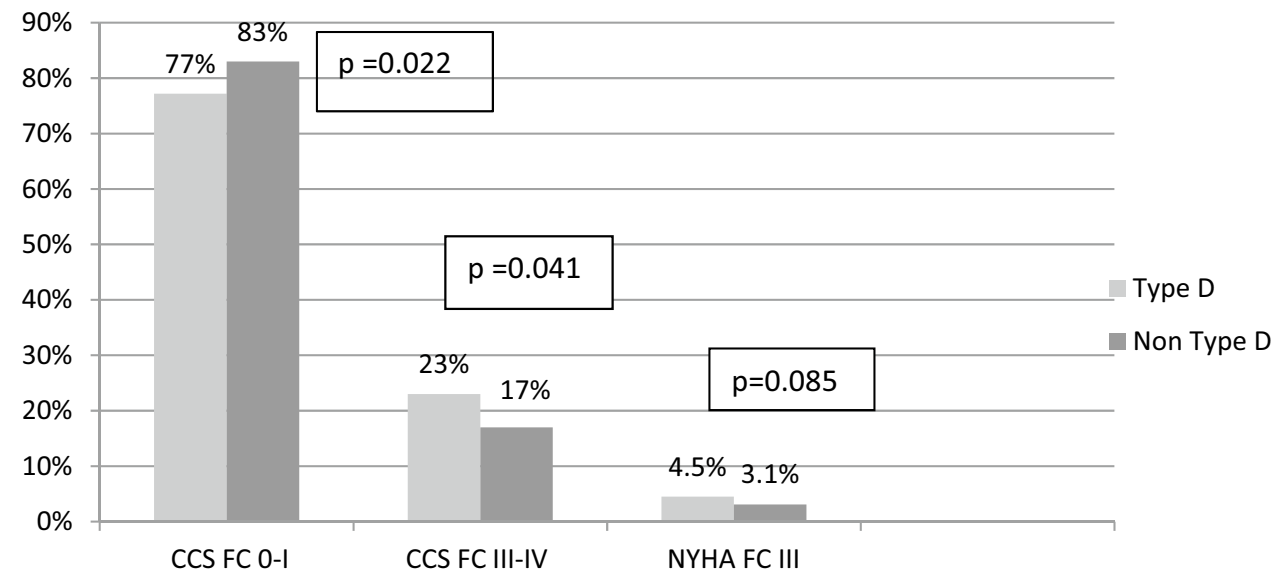


Fig. 3 Curves of freedom from the primary endpoint in patients with type $\mathrm{D}$ and without type $\mathrm{D}$ (Kaplan-Meier), $p=0.021$ between groups type $\mathrm{D}$ and without type D

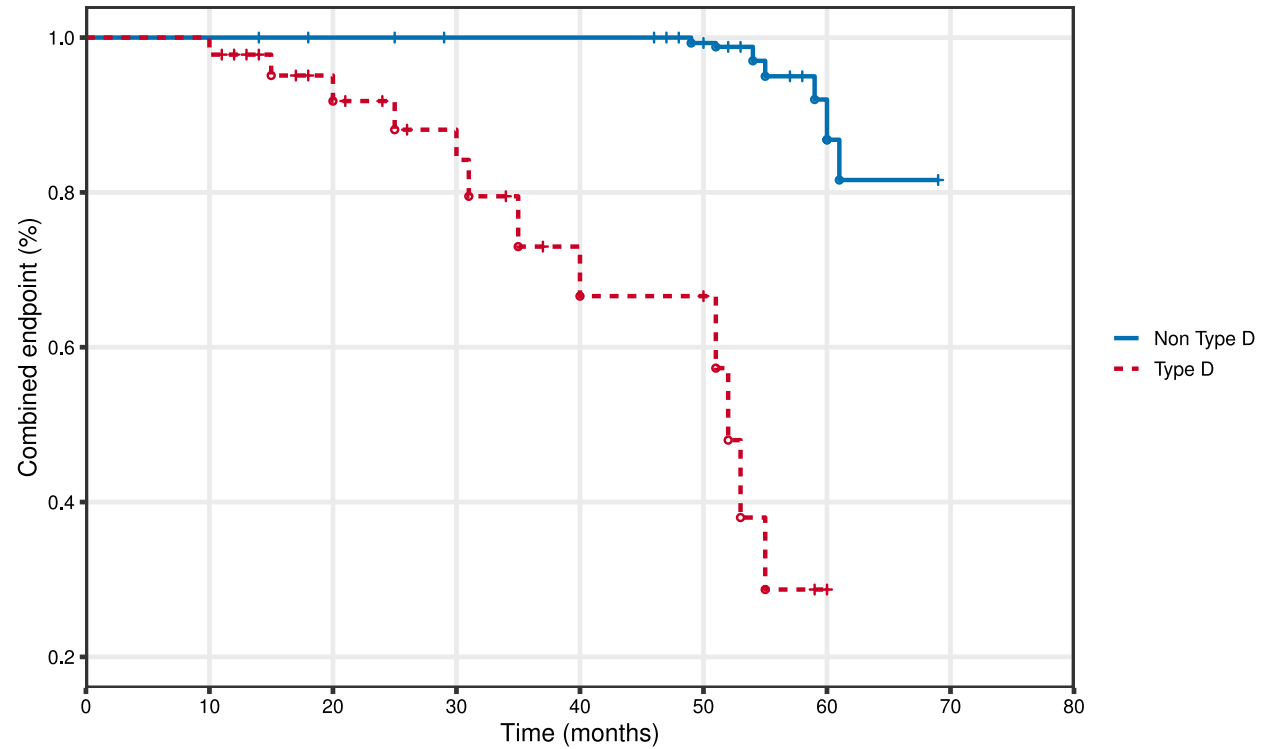

\section{Discussion}

This study shows that type D personality was associated with more frequent development of adverse outcomes in 5 years after CABG surgery as compared to patients without type D. Until now, not many studies have been devoted to the effect of personality type $\mathrm{D}$ on the results of coronary bypass surgery; however, they are generally consistent with the trend we have identified.

Previously, it has been demonstrated that type D patients are more likely to develop complications such as atrial fibrillation in the perioperative period [33] and reach a combined endpoint of fatal and nonfatal cardiac events during the year
[19]. It was also shown that type D personality negatively affects the quality of life in 6 months $[34,35]$ and at 1 year [36] after CABG. Our study shows that type D is both consistent in an individual for the observation time and has adverse prognostic value, such as more frequent occurrence of fatal and nonfatal cardiovascular events.

Earlier studies researched the influence of type D on the prognosis of indirect myocardial revascularization; however, conflicting results were obtained. Although Meyer et al. [37] found that type D personality did not affect the 5-year prognosis in patients after percutaneous coronary intervention (PCI) (although it should be noted that social inhibition, one of the components of type $\mathrm{D}$, was associated with more
Fig. 4 Curves of freedom from MACE in patients with type D and without type D (KaplanMeier), $p=.611$ between groups type $\mathrm{D}$ and without type $\mathrm{D}$

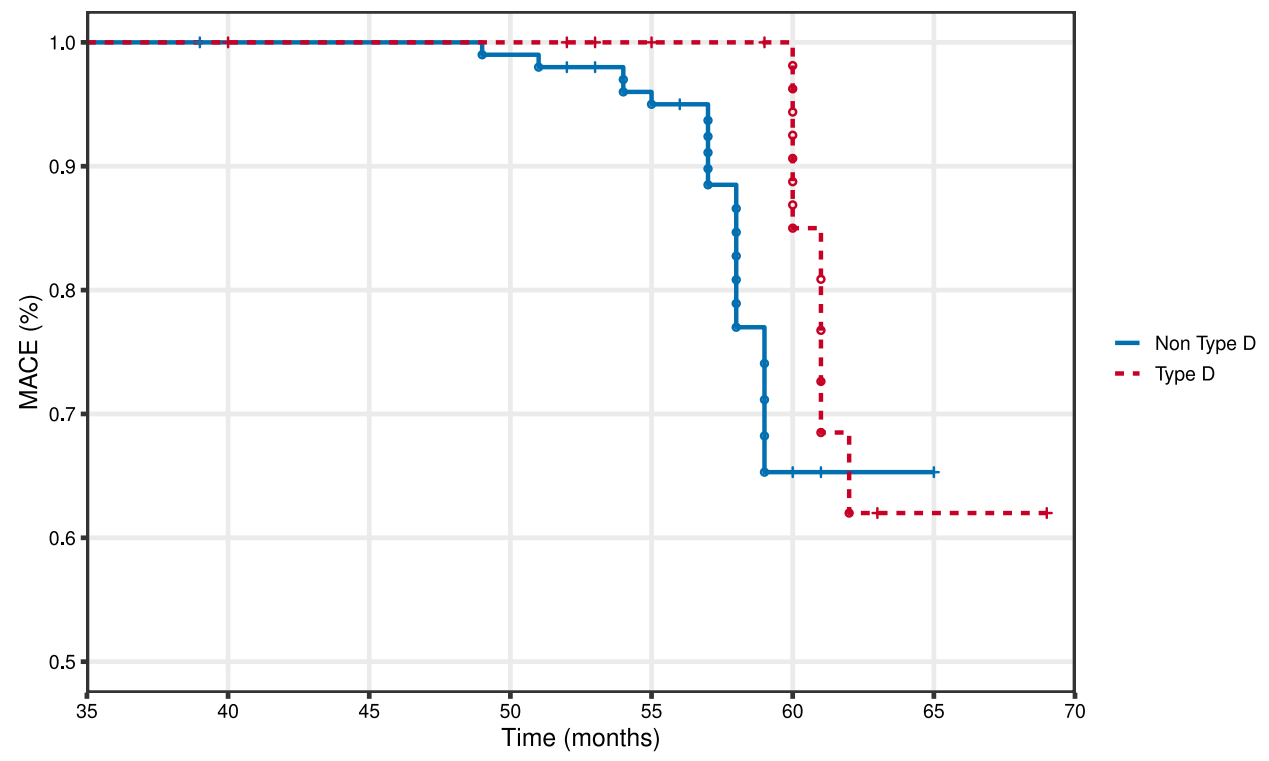


Table 4 Predictive parameters for combined endpoint (univariate and multivariate logistic regression analysis)

\begin{tabular}{|c|c|c|}
\hline Variables & OR $(95 \% \mathrm{CI})$ & $p$ value \\
\hline Age & $2.82(1.42-3.93)$ & 0.073 \\
\hline Sex & $1.61(1.13-2.16)$ & 0.161 \\
\hline Ejection fraction & $1.12(1.01-1.21)$ & 0.062 \\
\hline Body mass index & $1.47(0.89-2.45)$ & 0.261 \\
\hline Atrial fibrillation & $1.22(0.41-3.59)$ & 0.234 \\
\hline Smoking & $1.52(1.07-2.14)$ & 0.082 \\
\hline Hypertension & $1.57(0.32-7.60)$ & 0.242 \\
\hline Type D personality & $2.17(0.91-4.61)$ & 0.042 \\
\hline Hypercholesterolemia & $2.89(1.45-4.24)$ & 0.025 \\
\hline Previous stroke & $2.91(0.55-4.23)$ & 0.023 \\
\hline Diabetes mellitus & $2.92(1.07-8.8)$ & 0.041 \\
\hline Multifocal atherosclerosis & $3.60(0.95-11.34)$ & 0.011 \\
\hline \multicolumn{3}{|c|}{$\begin{array}{l}\text { Model 1. Predictive parameters for combined endpoint regardless of } \\
\text { gender and age }\end{array}$} \\
\hline Smoking & $1.02(1.01-2.11)$ & 0.025 \\
\hline Type D personality & $1.24(1.01-1.53)$ & 0.044 \\
\hline Atrial fibrillation & $2.12(0.75-6.02)$ & 0.018 \\
\hline Previous stroke & $3.15(0.35-5.22)$ & 0.021 \\
\hline Multifocal atherosclerosis & $3.21(2.02-6.14)$ & 0.023 \\
\hline Diabetes mellitus & $3.75(0.15-5.34)$ & 0.011 \\
\hline \multicolumn{3}{|c|}{$\begin{array}{l}\text { Model } 2 \text { Predictive parameters for combined endpoint, regardless of } \\
\text { gender, age, MI, and stroke }\end{array}$} \\
\hline Type D personality & $1.44(1.21-1.81)$ & 0.039 \\
\hline Diabetes mellitus & $3.25(1.25-6.24)$ & 0.021 \\
\hline Multifocal atherosclerosis & $3.61(1.32-6.24)$ & $\mathbf{0 . 0 3 3}$ \\
\hline
\end{tabular}

The entries in italics indicate borderline statistical significance of differences between groups $(p<0.1)$, the entries in bold indicate significant differences $(p<0.05)$ between groups

frequent development of nonfatal cardiovascular events), other studies show a negative effect of type $\mathrm{D}$ on prognosis $[18,38,39]$. There was no difference in mortality during the observation period (exactly like in our study), but presence of type D personality was associated with higher incidence of adverse cardiovascular events [38, 39]. Study by Wang et al. [25] demonstrated one of the possible mechanisms of adverse prognostic influence of type D personality: relationship was observed between type D and stent restenosis [25] found during routine coronary angiography 1 and 2 years after a coronary artery stenting. Optical coherence tomography shows that after PCI such patients develop excessive neointimal proliferation, neoatherosclerosis, and thin cap fibroatheromas in stent more frequently [40]. This development is facilitated by endothelial dysfunction [41] and elevated levels of proinflammatory cytokines [8], as well as other known biological phenomena typical for patients with type D [42]. Masdjedi K. et al. conducted an interesting analysis of patient preferences in the OPINION study [43], which showed that patients with type D personality prefer a coronary bypass surgery to PCI as the revascularization method more often compared to patients without type D. However, while such personal preferences may cause higher frequency of type D personality detected in patients undergoing $\mathrm{CABG}$, they cannot affect prognosis. Obviously, there is no substrate for neoatherosclerosis formation in patients after CABG because there are no stents, but other factors (endothelial dysfunction and subclinical inflammation) remain. Furthermore, the optical coherence tomography data in CAD patients shows independent association between type $\mathrm{D}$ personality and plaque vulnerability, thin cap fibroatheroma, rupture, and lipid arc [44]. It should be noted that known behavioral characteristics of patients with type $\mathrm{D}[12,45,46]$ contribute to the realization of adverse prognostic influence as well. For example, Son YJ et al. [47] showed that patients with type D personality are 5 times more likely to have low compliance to prescription drugs than patients without type D 3 months after PCI. In addition, multifocal atherosclerosis in patients with type $\mathrm{D}$ personality is detected more often, which is also associated with an unfavorable prognosis in patients undergoing CABG $[48,49]$, as confirmed in our study. We also found a trend toward more frequent detection of cardiovascular risk factors such as smoking and diabetes mellitus in type D. Despite the fact that differences with patients without type D did not reach statistical significance $(p=0.051$ and $p=0.056$, respectively), these risk factors also further contributed to a negative impact on the prognosis of personality type $\mathrm{D}$, as and more frequent detection of myocardial infarction and stroke in the history. In general, the above considerations are consistent with the identification of poor prognosis in patients after CABG with type D personality.

So, why did the previously conducted studies not always confirm this pattern, in fact, at one time the type D association with the influence on prognosis was doubted at all [15]? Firstly, significant heterogeneity of psychological traits among coronary heart disease patients is noted, considering not only selection of a subgroup with high levels of psychological distress, but also a subgroup with passive overcoming which is also associated with adverse behavioral reactions (in particular, infrequent participation in rehabilitation programs) [50]. Secondly, a further analysis showed significant heterogeneity of the influence of type D personality on prognosis in cardiac patients [20]. The reasons for this heterogeneity may include inconsistency in selecting endpoints (type $\mathrm{D}$ has a prognostic influence regarding cardiac complications, but is not known to affect noncardiac death) and age of patients (type $\mathrm{D}$ had no association with the prognosis in the older age). Thus, no influence of type $\mathrm{D}$ on the prognosis has been identified in patients with chronic heart failure, unlike the coronary patients [15]. Perhaps one of the reasons for this is that the cause of death in these patients often cannot be accurately determined [12]. It should be noted the ethnic 
and cultural differences can influence the predisposition to distress and various clinical parameters such as quality of life [51]. In part, this may be caused by different interpretations of life situations suggested in the questionnaire DS-14 $[52,53]$. This study showed that in other socio-economic, ethnic and geographical conditions, the prognostic value of personality type D may be more pronounced than in Germany and the Netherlands, although this fact requires further confirmation in other countries.

What are the clinical implications of this study? First, given the adverse effect of personality type $\mathrm{D}$ on the prognosis and quality of life after coronary artery bypass grafting, such patients should be identified in a timely manner, even at the stage of preoperative examination. This is quite in the spirit of the modern patient-centered approach. Second, these patients require targeted behavioral interventions both before surgery and during postoperative rehabilitation. Apparently, they should include the correction of excessive responses to stress (training in muscle relaxation, meditation, autogenic training, etc.). So far, the results of such attempts are inconsistent. Thus, an 8-week course of stress-limiting therapy did not reduce the number of patients with type D personality, despite a slight decrease in the NA and SI scores [54] (which is not surprising, given the stability of the type D construct over time). However, spending time in psychotherapeutic rehab groups gives patients the opportunity to understand the impact of psychological and biological risk factors, and to share responsibility for developing strategies to deal with daily stress. In this form, a personified approach to patient rehabilitation is most fully implemented [55].

Nevertheless, the task of future research is to develop an effective strategy for correcting the adverse effect of personality type $\mathrm{D}$ on the prognosis and quality of life of patients. Perhaps it will be a special therapeutic technique such as "positive emotional writing" [56] or improving the perception of illness [57], or overcoming inadequate coping strategies for stress [58]. The problem may be the reluctance of type D persons to seek help (fear of social interactions) [59]. Since for the effective work of the psychotherapist, it is necessary for the patient to address him with the presence of a problem, then such a personality trait of type $\mathrm{D}$ persons may present additional difficulties in behavioral therapy. However, staged psychotherapy to improve depressive symptoms was particularly helpful in CAD patients with Type D personality in the multicenter SPIRR-CAD study [60], suggesting promising research in this area.

\section{Study Limitations}

There are some limitations of this study which future research should address. First, the study was unable to identify the influence of susceptibility to psychological distress on the secondary endpoints (deaths, nonfatal MI, stroke). Perhaps this was due to the small number of such events during the observation period. Apparently, manifestation of such relationships requires a greater number of patients or conducting a meta-analysis. Second, we only used the dichotomous method of type D personality assessing, without using other approaches (assessment of 4 groups). We used the most convenient evaluation method, which showed clinical significance in our previous studies. Evaluation of the "type D effect" of using 4 groups is recognized as inappropriate in recent work [18]. In future, it is necessary to evaluate the influence of type D using the entire set of methods for its assessment, and such meta-analysis is already underway, including the data from this study. Third, in this study, the sample was largely male; given the paucity of women in the study, future research must also consider the generalizability of these findings in the context of sex differences. Finally, this study examined patients from one country (Russian Federation), so questions arise about the possibility of generalizing them to other regions. However, the results of this article confirm the initial results on the adverse prognostic effect of personality type $\mathrm{D}$ in other socio-economic conditions, which is an important addition to them.

\section{Conclusions}

Type D personality was detected in $22.4 \%$ of patients over 5 years after $C A B G$. Multifocal atherosclerosis and angina pectoris were more common in group with type $\mathrm{D}(p=.041)$. Presence of type D personality criteria at initial examination increased combined the relative odds of nonfatal and fatal events by 3.21 times over a long term period (OR 1.44, 95\% CI1.21-1.81, $p=0.039$ ). Type D patients had a greater risk for combined endpoint compared to non-type D patients. Obtained data indicates that it is reasonable to consider personality type while detecting patients at risk of development of stress induced cardiac complications after CABG in Russian population.

Supplementary Information The online version contains supplementary material available at https://doi.org/10.1007/s12529-021-09992-y.

\section{Declarations}

Ethical Approval The study has been approved by the local research ethics committee and has been performed in accordance with the ethical standards as laid down in the 1964 Declaration of Helsinki and its later amendments or comparable ethical standards.

Informed Consent Informed consent was obtained from all individual participants included in the study.

Conflict of Interest The authors declare no competing interests. 


\section{References}

1. Langberg EM, Dyhr L, Davidsen AS. Development of the concept of patient-centredness - A systematic review. Patient Educ Couns. 2019;102(7):1228-36.

2. Kim C, Hong SJ, Ahn CM, et al. Patient-centered decision-making of revascularization strategy for left main or multivessel coronary artery disease. Am J Cardiol. 2018;122(12):2005-13.

3. Denollet J, Rombouts H, Gillebert TC, Brutsaert DL, Sys SU, Stroobant N. Personality as independent predictor of long-term mortality in patients with coronary heart disease. The Lancet. 1996;347:417-21.

4. Denollet J. DS14: standard assessment of negative affectivity, social inhibition, and Type D personality. Psychosom Med. 2005;67(1):89-97.

5. Bunevicius A, Brozaitiene J, Staniute M, et al. Decreased physical effort, fatigue, and mental distress in patients with coronary artery disease: importance of personality-related differences. Int J Behav Med. 2014;21(2):240-7.

6. O'Dell KR, Masters KS, Spielmans GI, Maisto SA. Does type-D personality predict outcomes among patients with cardiovascular disease? A meta-analytic review. J Psychosom Res. 2011;71(4):199-206.

7. Bouwens E, van Lier F, Rouwet EV, Verhagen HJM, Stolker RJ, Hoeks SE. Type D personality and health-related quality of life in vascular surgery patients. Int J Behav Med. 2019;26(4):343-51.

8. van Dooren FE, Verhey FR, Pouwer F, et al. Association of Type $D$ personality with increased vulnerability to depression: Is there a role for inflammation or endothelial dysfunction? - The Maastricht Study. J Affect Disord. 2016;189:118-25.

9. Williams L, O'Connor RC, Grubb N, O'Carroll R. Type D personality predicts poor medication adherence in myocardial infarction patients. Psychol Health. 2011;26(6):703-12.

10. Wu JR, Moser DK. Type D personality predicts poor medication adherence in patients with heart failure in the USA. Int J Behav Med. 2014;21(5):833-42.

11. Schiffer AA, Denollet J, Widdershoven JW, Hendriks EH, Smith OR. Failure to consult for symptoms of heart failure in patients with a type-D personality. Heart. 2007;93(7):814-8.

12. Kupper N, Denollet J. Type D personality as a risk factor in coronary heart disease: A review of current evidence. CurrCardiol Rep. 2018;20(11):104.

13. Piepoli MF, Hoes AW, Agewall S, et al. 2016 European Guidelines on cardiovascular disease prevention in clinical practice: the Sixth Joint Task Force of the European Society of Cardiology and Other Societies on Cardiovascular Disease Prevention in Clinical Practice (constituted by representatives of 10 societies and by invited experts) Developed with the special contribution of the European Association for Cardiovascular Prevention \& Rehabilitation (EACPR). Eur Heart J. 2016;37(29):2315-81.

14. Pelle AJ, Pederson SS, Schiffer AA, Szabó B, Widdershoven JW, Denollet J. Psychological distress and mortality in systolic heart failure. Circ Heart Fail. 2010;3:261-7.

15. Coyne JC, Jaarsma T, Luttik ML, van Sonderen E, van Veldhuisen DJ, Sanderman R. Lack of prognostic value of type D personality for mortality in a large sample of heart failure patients. Psychosom Med. 2011;73(7):557-62.

16. Grande G, Romppel M, Vesper JM, Schubmann R, Glaesmer H, Herrmann-Lingen C. Type D personality and all-cause mortality in cardiac patients - data from a German cohort study. Psychosom Med. 2011;73(7):548-56.

17. Grande G, Romppel M, Barth J. Association between type D personality and prognosis in patients with cardiovascular diseases: a systematic review and meta-analysis. Ann Behav Med. 2012;43(3):299-310.
18. Dulfer K, Hazemeijer BAF, Van Dijk MR, et al. Prognostic value of type D personality for 10-year mortality and subjective health status in patients treated with percutaneous coronary intervention. J Psychosom Res. 2015;79:214-21.

19. Sumin AN, Raikh OI, Gaifulin RA, et al. Predisposition to psychological distress in patients after coronary bypass surgery: Relation to one year prognosis. Kardiologiia. 2015;55(10):76-82 (in Russian).

20. Kupper N, Denollet J. Explaining heterogeneity in the predictive value of type $\mathrm{D}$ personality for cardiac events and mortality. Int $\mathrm{J}$ Cardiol. 2016;224:119-24.

21. Smith TW. Toward a more systematic, cumulative and applicable science of personality and health: lessons from Type D personality. Psychosom Med. 2011;73:528-32.

22. Lodder P. Modeling synergy: How to assess a Type D personality effect. J Psychosom Res. 2020;132:109990.

23. Denollet J, Pedersen SS, Vrints CJ, Conraads VM. Predictive value of social inhibition and negative affectivity for cardiovascular events and mortality in patients with coronary artery disease: the Type D personality construct. Psychosom Med. 2013;75(9):873-81.

24. Wang Y, Zhao Z, Gao X, et al. Type D personality and coronary plaque vulnerability in patients with coronary artery disease: an optical coherence tomography study. Psychosom Med. 2016;78:583-92.

25. Wang Y, Liu G, Gao X, et al. Prognostic value of type D personality for in-stent restenosis in coronary artery disease patients treated with drug-eluting stent. Psychosom Med. 2018;80(1):95-102.

26. Williams L, O'Connor RC, Grubb NR, O'Carroll RE. Type D personality and threemonth psychosocial outcomes among patients post-myocardial infarction. J Psychosom Res. 2012;72:422-6.

27. Bousquet J, Anto JM, Iaccarino G, et al. ARIA group; Is diet partly responsible for differences in COVID-19 death rates between and within countries? Clin Transl Allergy. 2020;10:16.

28. Kupper N, Pedersen SS, Höfer S, Saner H, Oldridge N, Denollet J. Cross-cultural analysis of type D (distressed) personality in 6222 patients with ischemic heart disease: a study from the International HeartQoL Project. Int J Cardiol. 2013;166(2):327-33.

29. Pushkarev GS, Kuznetsov VA, Yaroslavskaya EI, Bessonov IS. Reliability and validity of russian version of DS14 score for ischemic heart disease patients. Russ J Cardiol. 2016;134(6):50-4 ((in Russian)).

30. Raykh OI, Sumin AN, Kokov AN, Indukaeva EV, Artamonova $\mathrm{GV}$. Association of type D personality and level of coronary artery calcification. J Psychosom Res. 2020;139:110265.

31. Sumin AN, Raih OI. Influence of Type D personality on adherence to treatment in cardiac patients. Kardiologiia. 2016;56(7):78-83 (in Russian).

32. Sumin AN, Raikh OI, Karpovich AV, Korok EV, Bezdenezhnykh $\mathrm{AV}$, Bokhan YaE, et al. Personality types in patients with atherosclerosis of different localization: prevalence and clinical features. Clin Med. 2012;4:43-9 (in Russian).

33. Kelpis TG, Anastasiadis K, Nimatoudis I, Kelpi MG, Hadjimiltiades S, Papakonstantinou C. Prevalence of "distressed" personality in patients with coronary artery disease and its correlation with morbidity after coronary surgery. Hellenic J Cardiol. 2013;54(5):362-7.

34. Dannemann S, Matschke K, Einsle F, et al. Is type-D a stable construct? An examination of type-D personality in patients before and after cardiac surgery. J Psychosom Res. 2010;69(2):101-9.

35. Middel B, El Baz N, Pedersen SS, van Dijk JP, Wynia K, Reijneveld SA. Decline in health-related quality of life 6 months after coronary artery bypass graft surgery: the influence of anxiety, depression, and personality traits. J Cardiovasc Nurs. 2014;29(6):544-54.

36. Al-Ruzzeh S, Athanasiou T, Mangoush O, et al. Predictors of poor mid-term health related quality of life after primary isolated coronary artery bypass grafting surgery. Heart. 2005;91:1557-62.

37. Meyer T, Hussein S, Lange HW, Herrmann-Lingen C. Type D personality is unrelated to major adverse cardiovascular events 
in patients with coronary artery disease treated by intracoronary stenting. Ann Behav Med. 2014;48(2):156-62.

38. Du J, Zhang D, Yin Y, et al. The personality and psychological stress predict major adverse cardiovascular events in patients with coronary heart disease after percutaneous coronary intervention for five years. Medicine (Baltimore). 2016;95(15):e3364.

39. Leu HB, Yin WH, Tseng WK, et al. Impact of type D personality on clinical outcomes in Asian patients with stable coronary artery disease. J Formos Med Assoc. 2019;118(3):721-9.

40. Lee $\mathrm{R}, \mathrm{Yu} \mathrm{H}, \mathrm{Gao} \mathrm{X}$, et al. The negative affectivity dimension of Type D personality is associated with in-stent neoatherosclerosis in coronary patients with percutaneous coronary intervention: An optical coherence tomography study. J Psychosom Res. 2019;120:20-8.

41. Denollet J, van Felius RA, Lodder P, et al. Predictive value of Type D personality for impaired endothelial function in patients with coronary artery disease. Int J Cardiol. 2018;259:205-10.

42. Denollet J, Kupper N. Stress and the heart: the role of type D personality in personalized care. Eur Heart J. 2015;36(28):1783-5.

43. Masdjedi K, Daemen J, Diletti R, et al. A case-vignette based assessment of patient's perspective on coronary revascularization strategies, the OPINION study. J Cardiol. 2018;72(2):149-54.

44. Cheng F, Lin P, Wang Y, et al. Type D personality and coronary atherosclerotic plaque vulnerability: The potential mediating effect of health behavior. J Psychosom Res. 2018;108:54-60.

45. Mommersteeg PM, Kupper N, Denollet J. Type D personality is associated with increased metabolic syndrome prevalence and an unhealthy lifestyle in a cross-sectional Dutch community sample. BMC Public Health. 2010;10:714.

46. Wiencierz S, Williams L. Type D personality and physical inactivity: the mediating effects of low self-efficacy. J Health Psychol. 2017;22(8):1025-34.

47. Son YJ, Lee K, Morisky DE, Kim BH. Impacts of Type D Personality and Depression, Alone and in Combination, on Medication Non-Adherence Following Percutaneous Coronary Intervention. Int J Environ Res Public Health. 2018;15(10).

48. Morikami Y, Natsuaki M, Morimoto T, et al. Impact of polyvascular disease on clinical outcomes in patients undergoing coronary revascularization: an observation from the CREDO-Kyoto Registry Cohort-2. Atherosclerosis. 2013;228(2):426-31.

49. Gajfulin RA, Sumin AN, Ivanov SV, Barbarash LS. Survival after surgical treatment of multifocal atherosclerosis in different age groups. Complex Issues Cardiovasc Dis. 2017;2:6-18 (in Russian).
50. Van Montfort E, Kupper N, Widdershoven J, Denollet J. Personcentered analysis of psychological traits to explain heterogeneity in patient-reported outcomes of coronary artery disease- the THORESCI study. J Affect Disord. 2018;236:14-22.

51. El-Baz N, Ondusova D, Studencan M, et al. Differences between Slovak and Dutch patients scheduled for coronary artery bypass graft surgery regarding clinical and psychosocial predictors of physical and mental health-related quality of life. Eur J Cardiovasc Nurs. 2018;17(4):324-35.

52. Lim HE, Lee MS, Ko YH, et al. Assessment of the type D personality construct in the Korean population: A validation study of the Korean DS14. J Korean Med Sci. 2011;26(1):116-23.

53. Pushkarev G, Kuznetsov V, Yaroslavskaya E, Bessonov I. Social support for patients with coronary artery disease after percutaneous coronary intervention. J Psychosom Res. 2019;119:74-8.

54. Nyklíček I, van Beugen S, Denollet J. Effects of mindfulnessbased stress reduction on distressed (type D) personality traits: a randomized controlled trial. J Behav Med. 2013;36(4):361-70.

55. Pah AM, Buleu NF, Tudor A, et al. Evaluation of psychological stress parameters in coronary patients by three different questionnaires as pre-requisite for comprehensive rehabilitation. Brain Sci. 2020;10(5):316.

56. Smith MA, Thompson A, Hall LJ, et al. The physical and psychological health benefits of positive emotional writing: Investigating the moderating role of Type $\mathrm{D}$ (distressed) personality. Br J Health Psychol. 2018;23(4):857-71.

57. Kwon M, Kang J. Mediating effect of illness perception on the relationship between Type D personality and health behaviors among coronary artery disease patients. Health Psychol Open. 2018;5(2):2055102918817228.

58. Martin LA, Doster JA, Critelli JW, et al. The 'distressed' personality, coping and cardiovascular risk. Stress Health. 2011;27:64-72.

59. Arrebola-Moreno M, Petrova D, Garrido D, et al. Psychosocial markers of pre-hospital decision delay and psychological distress in acute coronary syndrome patients. Br J Health Psychol. 2020;25(2):305-23.

60. Herrmann-Lingen C, Beutel ME, Bosbach A, et al. A Stepwise Psychotherapy Intervention for Reducing Risk in Coronary Artery Disease (SPIRR-CAD): results of an observer-blinded, multicenter, randomized trial in depressed patients with coronary artery disease. Psychosom Med. 2016;78(6):704-15.

Publisher's Note Springer Nature remains neutral with regard to jurisdictional claims in published maps and institutional affiliations. 